\title{
Historical Walk: The HRP Special Programme and Infertility
}

\author{
Sheryl Ziemin van der Poel \\ HRP Special Programme, Geneva, Switzerland
}

\begin{abstract}
Background/Aims: The HRP Special Programme (HRP) began addressing 'reproduction' problems (infertility) at the same time as 'fertility regulation' (contraception). Methods: This report is based upon data collected from official HRP Scientific and Technical Reports. Results: In the 1970s, HRP supported research on human and nonhuman primate models to address the basic biology of reproduction. Importantly, however, it was the multicountry clinical research studies sponsored by HRP during the 1970s and 1980s which led to the identification of not only the large burden of disease but also global patterns of causation of infertility. The next decade saw the development of WHO guidelines and manuals for diagnosis, management, and treatment of infertile women and men, with HRP-sponsored operations research focused on adaptation within primary health care settings. Consensus consultations held during 2001 through 2011 resulted in recommendations to stakeholders, as well as the identification of barriers and inequities in access to infertility care services. Conclusion: With renewed focus on infertility through the current development of revised guidelines and manuals, and the desire to support critical clinical research protocols, including adaptation and implementation research for management and monitoring tools being developed through international partners, HRP will continue to support future success stories in family, maternal, child, and reproductive health.

Copyright $\odot 2012$ S. Karger AG, Basel
\end{abstract}

\section{Reproduction Matters}

Interventions which assist to resolve involuntary infertility have not only been medically and scientifically innovative, but they have also revolutionized concepts of generational identity, family, and human reproductive potential. It is the choice of each individual, within their own sense of conscience, to determine the size of their family unit and the timing of when to have children. The 18th World Health Assembly [1] recognized this right to address human reproductive health needs, from the concepts of fertility control as well as to address fertility problems, which resulted in the establishment of a World Health Organization (WHO) unit dedicated to human reproduction. Planning a family impinges on issues associated with social and economic development, as recognized by delegates from the 21st World Health Assembly [2] who also identified the need for every individual to have the opportunity to obtain information and advice on problems connected with forming a family unit. Taking up these recommendations, a WHO Scientific Group on the Biological Components of Human Reproduction was established, and it stated that in order to understand fertility problems research was required to perform a needs assessment and understand the global burden of infertility [3]. In addition, the Expanded (later Special) Programme of Research, Development and Research

\section{KARGER}

Fax +4161306 1234

E-Mail karger@karger.ch

www.karger.com
(C) 2012 S. Karger AG, Basel

0378-7346/12/0743-0218\$38.00/0

Accessible online at:

www.karger.com/goi
Dr. Sheryl Ziemin van der Poel HRP Special Programme CH-1211 Geneva (Switzerland)

E-Mail vanderpoels@who.int 
Training in Human Reproduction (Human Reproduction Programme, HRP, or the Programme) was established in 1972 [4]. The research Programme, now into its fifth decade, began with a focus on fertility regulation (contraception) but also addressed fertility problems (infertility). Indeed, HRP involvement in infertility began during the Programme's first decade of existence!

One of the first symposia (1971) sponsored by (the Expanded) HRP brought together experts in human and nonhuman primate (NHP) reproduction research, in order to address NHPs as a model for human reproductive research as well as to address problems arising in the reproduction of NHPs themselves. This WHO symposium included in its report two papers presented by the Nobel Laureate Robert G. Edwards, which defined his early work on human embryo in vitro culture [5]. A Scientific Group on the Epidemiology of Infertility was formed and met in 1975, which resulted in a Technical Report [6] which made the following specific recommendations to the Programme: to assist in global epidemiological and etiological research into infertility, and to provide a means by which such research can be coordinated and results compared between different countries worldwide. The Scientific Group defined infertility and included in its definition women who had what was described at the time as 'pregnancy wastage' or spontaneous abortions/early miscarriages, and who were unable to become pregnant, unable to bring a child to term, and/or unable to have a live birth.

\section{Addressing an Unfulfilled Child Wish}

In 1979, the Task Force on the Diagnosis and Treatment of Infertility was established to meet the Scientific Group's recommendations endorsed by the Programme. The first consultation was a regional workshop held in collaboration with the Kenya Obstetrical and Gynaecological Society in Nairobi, and opened by the assistant minister of health of Kenya. Specifically, dedicated to the 'Year of the Child' with a focus on those 'missing' children for couples eager but unable to have a child, it included a discussion of the linkage between the periconceptional period and the health of the mother and the future child [7]. The Task Force initiated its first epidemiological studies in Brazil, China, and India and found that the determination of not only the prevalence but also the causality of infertility, in both men and women, was required to understand a public health needs assessment and burden. However, as a result of the Declaration of Alma Ata [8], the role of the WHO had begun to shift to-

The HRP Special Programme and Infertility ward 'promotion of primary health care, with the adaptation and transfer of available technologies for use in primary health care'. The Task Force debated but made a conscious decision to first adopt a tertiary care level approach for infertility because little was known concerning the causes of fertility problems resulting in infertility, especially in developing countries. Thus, the Programme supported tertiary level clinical research studies initiated in Armenia, Benin, Brazil, Cameroon, China, India, Pakistan, Tanzania, Thailand, and Viet Nam. On close review of outcome data, the Task Force realized that a highly systematic, standardized method of diagnostic procedures using identical definitions was critical during the implementation of any future studies in order to improve precision in diagnosis, assessment of management strategies, and ultimately to confidently compare patterns from different geographical areas worldwide.

A multicountry, multicenter clinical research protocol was implemented in collaboration with and with the support of the Programme within 33 centers in 25 (10 developed and 15 developing) countries between 1979 and 1984 - and data on more than 5,800 infertile couples was collected. The following epidemiological definition was used: the inability of a woman, man, or couple, after a period of 2 years of exposure, to become pregnant or maintain a pregnancy. Despite the rigor of the protocol, because the couples evaluated were only those who were actively seeking care (those who were not seeking care, who feared diagnosis and stigma, or instead changed partners or were in fear of losing their partner, or determined infertility a fate and not a disease or disability, etc., were not counted), the prevalence rates were, and remain, a difficult task to estimate accurately. The Task Force extrapolated that a minimum of 80 million couples worldwide suffer from involuntary infertility. Also, there was great variability: 'primary infertility (no previous pregnancy) rates ranged from 3 to over $30 \%$ in low- and highrisk countries, and secondary infertility (having had a previous pregnancy) rates were in general double the primary infertility rates'. Specifically noted [9] was that key patterns had emerged: the centers from Africa reported a pattern of infertility which was different from those in other developing regions or developed countries. African couples 'were found to be more likely to have secondary infertility of a longer duration, a history of pregnancy complications and/or sexually transmitted infections or reproductive tract infections (STIs/RTIs), and a diagnosis of bilateral tubal occlusion or pelvic adhesions'. In men, in sub-Saharan African centers as well as those in Latin America, data showed that accessory gland infection 


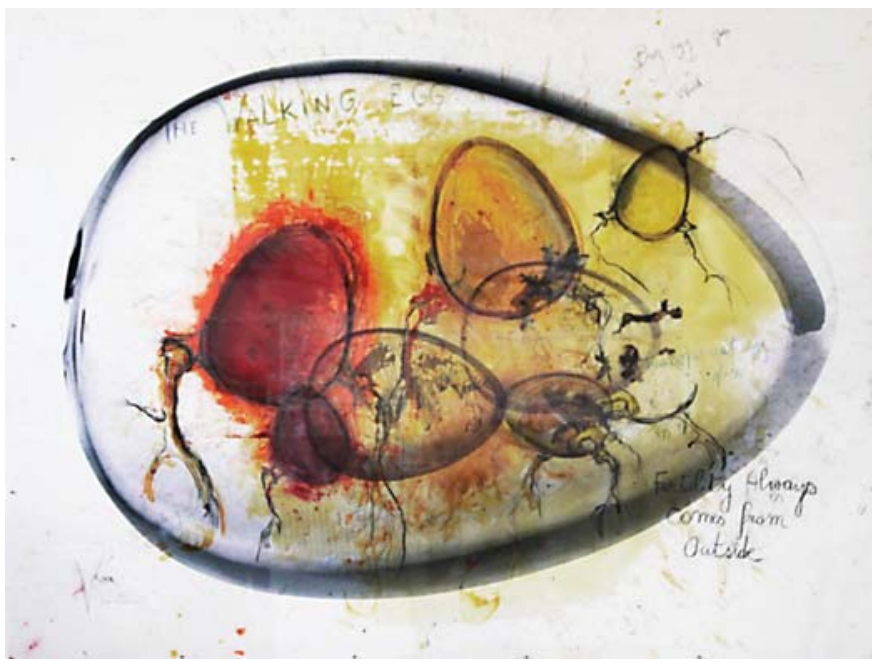

Fig. 1. Fertility always comes from outside, by Koen Vanmechelen, donated to the HRP Special Programme by The Walking Egg, a nonprofit organization.

rates were higher in comparison to other global regions; however, this regional distribution of diagnosis for men did not show the same dramatic regional variation as had been shown for African infertile women (but it did show that the majority of men at all research sites had idiopathic infertility). Despite the inability to determine if STIs/ RTIs caused the infertile state or were contracted as a result of unprotected sex to resolve an underlying infertile state, it was possible to postulate that STIs/RTIs could play a significant role - e.g. resulting in bilateral tubal occlusion or obstructive azoospermia resulting in infertility. The following recommendation was made: 'In African countries, in addition to management and treatment of those with infertility, it was clear that addressing prevention of infertility at the primary care level would be a critical approach to reducing the burden as evidenced by the etiology in women' [9]. Despite its acknowledged confounding issues and shortcomings, a multicountry, multicenter clinical research study of this scale and performed with such rigor has never been attempted since, and thus the results generated remain significant for the understanding of the global burden and causality patterns for infertility.

In light of the key patterns identified, the Programme endorsed the Task Force-recommended research agenda tailored to the African region, which would include training and implementation for 'the prevention of infertility at the primary health care level, which was based on the trial use of guidelines for the diagnosis and treatment of pelvic inflammatory disease (PID) and STIs/ RTIs, and with increased training in hygienic practices for maternal care: safe deliveries and/or abortions'. An assessment of the health services' ability to implement appropriate guidelines was critical (scaling-up capability), and further, prospective research was envisioned which could identify the effects that such changes within health services would have on reproductive health outcomes, provider and client perspectives, and specifically identifying outcome measures addressing infertility, as well as long-term women's and children's health. The Programme began to sponsor social science research, for example in Sierra Leone (in 1986) and in Kenya (in 1987) and later in several other African countries, in order to explore perceptions toward and understanding of infertility by primary and community health care workers.

\section{Prevention}

Gradually, the emphasis and priority on infertility within the Programme began to change. This was made evident in 1987 in the WHO review 'Infections, pregnancies and infertility: perspectives on prevention' [10], which stated that 'In the developing world, programs directed to sexually active persons to diagnose and treat infections ... may have a greater yield in preventing STI/RTI sequelae than current efforts directed to repair the damage' from STIs/RTIs, complications from child birth, and unsafe practices. It is also important to note that a significant decline in primary infertility in sub-Saharan Africa from the late 1950 s and early 1960 s to the last half of the 20th century 'had been postulated to be a result of the introduction of antibiotics for and awareness campaigns concerning STIs' [11]. The Programme further concluded that access to safe abortion practices and concentration on resolving complications resulting from childbirth would also help to prevent infertility, especially in developed countries and countries in the Asian region. Thus, at that time, the Programme placed a greater priority on 'prevention' as a key strategy for addressing the global burden of infertility, and significantly less prioritization on addressing or sponsoring research to address innovations and support for diagnosis, management, or affordable treatment for those already infertile. 


\section{Nobel Prize Innovation}

The WHO Scientific Group on Developments in Fertility Control recommended in the WHO Technical Report Series 424 [3] that in vitro systems suitable for the study of fertilization be researched and developed (for contraceptive innovations). In the 1980s, significant developments outside of the Programme helped to answer this recommendation. A ground-breaking innovation proved successful, with the first live human birth in 1978, which helped to resolve infertile couples' fertility problems through the use of in vitro manipulation of human gametes with embryonic extracorporeal development and transfer. The Nobel Prize in Physiology or Medicine in 2010 was awarded to Robert G. Edwards in recognition of his development (together with Patrick C. Steptoe, who died in 1988) of in vitro fertilization (IVF) innovative therapies [12].

Significantly, especially for developing countries with a high burden of infertility due to bilateral tubal occlusion, this innovation was an obvious treatment strategy since IVF allows the ability to bypass occluded tubes in women when tubal surgery has failed or is either useless or impossible to perform. A study in Lagos, Nigeria, looked at the etiological and sociomedical characteristics of infertility in 250 infertile couples and found that 'tubal disease was the commonest factor, being the sole factor in $24 \%$ and a factor in $41 \%$ of couples. ... Most tubes were usually so badly damaged that they were unlikely to be restored to normal function by any form of tubal surgery' [13]. Thus, the infertile with tubal disease were the most difficult to treat, especially without access to IVF technologies.

A Symposium on the Diagnosis and Treatment of Infertility sponsored by the Programme in 1985, and in collaboration with the (then) USSR and Armenia SSR Ministries of Health, was held at the Moscow Centre for $\mathrm{Hu}-$ man Reproduction in the All-Union Research Centre for Maternal and Child Health Care [14]. It became clear from the meeting recommendations to the Programme that guidelines for aspects of infertility diagnosis and management were needed, with a requirement to address technical aspects of IVF (including laboratory practices and personnel, and treatment modalities, as well as to address monitoring outcomes - including maternal, newborn, and child outcomes). A multidisciplinary Scientific Group was convened by the Programme, resulting in the development of technical guidelines covering infertility and IVF, published in 1992 [15].

Bringing together the outcomes generated from the multicountry study, investigations, guidelines, and other meeting reports, the WHO Manual for the Investigation and Diagnosis of the Infertile Couple was written and then published in 1993 [16] and has since been translated into 7 languages. The development of the Manual in infertility coincided with the 3rd revision of the WHO Laboratory Manual for the Examination of Human Semen and Sperm-Cervical Mucus Interaction [17] whose clear mandate had been to support research for new innovative approaches for contraception. However, at the country level, the analysis not only became important for fertile men but was also used to evaluate the semen of men in involuntarily infertile relationships.

\section{Strengthening Capacity and Adaptation at the Country Level}

During the late 1990s, the field of reproductive medicine addressing men had significantly changed since the Manual for the infertile couple had been written. Therefore, the Programme supported the development of a separate Manual addressing the infertile male [18], coinciding with the 4th revision of the WHO Laboratory Semen and Sperm-Mucus Manual [19]. From 1997 through 2008, the Programme disseminated the 3rd and 4th editions of the WHO Laboratory Semen Manual by providing technical and financial support to collaborating centers in Africa conducting 'semenology' laboratory training workshops, which resulted in the development of an adapted semen quality control program and the establishment of satellite andrology laboratories throughout the sub-Saharan region.

Although initially the Task Force had begun its work at the level of tertiary care, because of the development of the technical Guidelines and clinical Manuals, it had become possible for the Programme to support small operational research projects to address adaptation and barriers to scaling up for more affordable and simplified protocols within local settings. For example, investigators in Cuba modified and simplified the clinical Manual to their setting and in 1996 published results showing that $20 \%$ of their infertile couples could be managed at the Cuban primary health care level [20]. Notably, within the Maternal and Child Health Institute in Ulaanbaatar, Mongolia, a simplified management protocol of the infertile couple guidelines which did not include hormone estimations or laparoscopy was tested. For example, 'semen analysis looked only at estimates of the number/percent of progressively motile sperm without addressing morphology; hormone estimations and laparoscopy were not 
performed, and ovulatory disorders were treated empirically with clomiphene and/or bromcryptine. Unpublished data provided to the WHO showed that the pregnancy rates were $40-60 \%$, which were comparable to tertiary health care approaches'.

Infertility care services (and not prevention) were highly ranked as a priority area to be addressed along with family planning at a 'Joint South African and WHO Reproductive Task Force assessment of family planning services' [21]. 'Approximately 25\% of all patients seen in the gynecology outpatient department at Groote Schuur hospital in 1993/1994 presented with infertility as their primary complaint. In addition, the medical workload in terms of patient visits in the various clinics in the Reproductive Medicine (infertility) Unit of Groote Schuur Hospital exceeded that in the general gynecological units and comprised about $55 \%$ of the doctor consultations in the outpatient clinic' [11]. Recognizing that a disproportionately high number of couples required care, at the 2 nd Conference on Reproductive Health Priorities in 1995 a strategy was presented by South Africa for screening participants suitable for infertility counseling, treatment, or referral at the primary and secondary health care levels [22].

As part of UNFPA funding to the WHO Maternal and Child Health Unit, a document was commissioned to draft guidelines directed to the primary health care level with appropriate referral systems identified. However, these activities associated with infertility occurred during a transition period, and by 1998 the Programme had merged with the WHO Division of Reproductive Health, forming the Department of Reproductive Health and Research (RHR). As a result of the merger, there was a change in priorities and a new vision for family planning, and thus the primary care document for health workers on infertility, which required additional field testing and adaptation, did not remain a priority and the Infertility Task Force itself was closed down.

\section{Reproductive Rights, Ethics, and the Psychosocial and Economic Impact of Infertility}

The reproductive rights of individuals were defined by consensus at the International Conference on Population and Development (1994) in Cairo, Egypt. 'These (reproductive) rights, rest on the recognition of the basic right of all couples and individuals to decide freely and responsibly on the number, spacing, and timing of their children, and to have the information and means to do so, and the right to attain the highest standard of sexual and reproductive health. It also includes their right to make decisions concerning reproduction free of discrimination, coercion, and violence'. 'Means to do so' should include access to a quality standard of care and, in this light, concerns had arisen among UN Member States about various aspects of the application of 'assisted conception technologies' for infertile couples [23]. During the 52nd World Health Assembly (1999), the WHO was requested to review recent developments in the field of 'assisted conception' along with their social and ethical implications.

In response, the Programme held a meeting in 2001 within which participants presented their independent research addressing the psychosocial, gender, ethical, and equity issues associated with infertile couples and infertility care interventions. The meeting report [24] included a glossary of definitions prepared by the International Committee on Monitoring Assisted Reproductive Technologies (ICMART). The resultant consensus 87 recommendations strongly emphasized that the 'psychosocial and socioeconomic impact' of childlessness was significantly greater on individuals, couples, and communities from settings where access to diagnosis and affordable treatments were weakest or nonexistent. The particular identification of the need for access to affordable treatments in developing countries, as well as the importance of harmonized data collection on medical aspects of the couples together with the assisted reproductive practices utilized at national and international levels were highlighted. In addition to editing and releasing a meeting manuscript of research articles, the Programme supported advocacy articles, e.g. one specifically asking if the public health systems have yet begun to care about those who have become involuntarily infertile [25].

In response to an additional request, this time from the Regional Advisory Panel for Africa, a joint AFRO and EMRO regional workshop was held in Nairobi in 2002 in order to address the 'management of infertility for the African and Eastern Mediterranean regions'. Three key recommendations for these regions were (paraphrased): services for the prevention and management of unsafe abortions, traditional delivery practices, and STIs/RTIs and their complications, including infertility, should be addressed and provided at all levels of care; practice guides and algorithms for primary care should be completed and field tested, and, because surgi$\mathrm{cal} /$ medical treatment of infertility due to tubal damage is commonly unsuccessful, with recognition of limited resources, lower cost protocols for assisted conception 
should be considered for referral centers throughout the two regions [26].

Clinical research studies (incorporating a diagnosis of infertility with adjustments for evaluating prevalence values) were no longer being prioritized or supported by the Programme. However, the Programme together with Opinion Research Cooperation (ORC) Macro supported an analysis of Demographic and Health Survey (DHS) data, which resulted in an updated estimate on the burden of infertility in developing countries. Although the clinical etiology and thus the causation of infertility were unable to be determined, differentials addressing regional, socioeconomic, and other demographics were able to be reported. Using data covering the period of 1995-2000, and using a demographic definition of 5-year exposure to the risk of pregnancy without $a$ live birth, the WHO reported that $2.5 \%$ of couples (18 million) were estimated to experience primary involuntary infertility, with $25 \%$ of couples experiencing secondary infertility (168 million). This resulted in an estimated overall burden of 186 million couples of reproductive age (similar in burden to the high unmet need for family planning/contraception) in developing countries [27].

\section{The ART and Science of Resolving a Child Wish}

The WHO developed a Reproductive Health Strategy [4] to accelerate progress toward achievement of the Millennium Development Goals (MDGs) by attainment of the highest achievable standard for universal access to reproductive health. In September 2007, the UN General Assembly integrated the target MDG 5b 'achieve, by 2015, universal access to reproductive health'. Of the five core aspects of the Strategy, one addresses infertility: 'providing high-quality services for family planning, including infertility services' [28]. In December of that same year, the Programme participated in a conference in Arusha, Tanzania, supported by the European Society for Human Reproduction and Embryology (ESHRE) Special Task Force on 'Developing Countries and Infertility' [29]. The objective of this meeting, currently defined as the 'Arusha Initiative', was summed up by the coordinator of the Task Force as follows: 'After a fascinating period of almost 30 years of IVF and 15 years of ICSI (intracytoplasmic sperm injection), we must admit that only a small part of the world population benefits from these new reproductive technologies. Time has come to give adequate attention to the issue of infertility in developing coun-

The HRP Special Programme and Infertility tries.' In addition to convening a broad audience (policy makers, ministries, patient advocates, scientific and clinical researchers), presentations were given and included a Belgian artist. Innovatively transecting the fields of visual art and 'ART' by addressing reproduction, crossbreeding, and reproductive fitness, the meeting provided a human and earth view of reproduction and its impact on health.

Requests to the Programme had been made to update and revise the ICMART glossary of ART terms [30], and efforts and calls to determine affordable solutions for access to infertility care worldwide were increasing. Thus, a WHO consultation in collaboration with ICMART, the International Federation of Fertility Societies (IFFS), and the Low Cost IVF Foundation was convened at the WHO in 2008. A broad audience (including societies, editors of major journals addressing the field, patient advocates) representing over 80 countries was in attendance. Consensus was reached on an expanded number of terms and definitions [31] and, as stated, 'The need for standard definitions is critical for benchmarking the outcomes of assisted reproductive technology procedures, at both a national and an international level ... standardization is necessary so that monitoring of efficacy, safety, and quality of procedures and multinational research can be undertaken'. The glossary definitions are currently being assessed for integration into the ICD10 revision [32] and have, to date, been translated into 6 languages, including Dutch [33]. A pre-meeting and the second half of the consultation reviewed the status of existing WHO tools for providers which address infertility prevention, diagnosis, and management at primary, secondary, and tertiary levels of care, including the draft guidance for primary care health care workers. Meeting recommendations were made directly to the Programme through its advisory bodies, with requests for revitalization of the basic and clinical research in the area of infertility, especially within low-income settings, and to begin to develop WHO clinical guidelines and recommendations for infertility diagnosis, management, and treatment that would drive and provide evidence for policy and programming documents or provider/patient tools. Following the meeting, the Programme together with regional and country office support or with direct support from a fertility society, such as ESHRE, the International Federation of Gynecology and Obstetrics (FIGO), the American Society of Reproductive Medicine (ASRM), the International Society for in vitro Fertilization (ISIVF), the International Society for Mild Approaches to Assisted Reproduction (ISMAAR), and the IFFS, as well as ICMART, supported 
WHO technical presentations resulting from the 2004 DHS and the 2008 meeting outcomes and ethical issues in order to promote awareness of the public health issue of infertility.

\section{Barriers to Universal Access to Infertility Care}

With access to antiretroviral therapy and an increase in successful interventions to decrease parent-to-child transmission of HIV, the Programme, in collaboration with the Harvard Center for Population and Development Studies and the Program on International Health and Human Rights, held a conference in 2010 to address the pregnancy intentions of HIV-positive women [34]. The report states that: Access to assisted reproductive technologies ... may significantly influence HIV-positive women's desire and ability to bear children: they may also improve HIV-positive women's fertility while reducing the risk of horizontal transmission to HIV-negative male partners. Such technologies, however, are not universally available and are unevenly accessible or commonly lacking in low-income countries ... Even when available, the idea that ART could be used by women who are HIV positive appears to be strongly opposed. This is an area where stigma and discrimination are highly manifest.'

In December of that same year, two articles appeared in the WHO Bulletin $[35,36]$. When serving indigenous populations, due to practices to resolve a fertility problem which have the potential to increase HIV transmission, it was recognized that 'couples are looking for safer solutions to overcome infertility'. Within the WHO Bulletin advocacy piece, a prominent expert resolutely states: 'provision of the service falls significantly short in developing countries'. The article continues, stating that: despite their importance, infertility prevention and care often remain neglected public health issues, or at least they rank low on the priority list, especially for low-income countries that are under population pressure. Low fertility is becoming common worldwide, particularly in aging populations and many urban settings, where women are having their first babies at older ages. Also, patient advocacy groups recognize the conundrum of 'barrenness amid plenty' - referring to the fact that infertility is often most prevalent where fertility rates, and thus contraceptive targeting campaigns, are high. Mahmoud Fathalla, previous director of the Programme, provided words of understanding and wisdom: 'In a world that needs vigorous control of population growth, concerns about infertility may seem odd, but the adoption of a small family norm makes the issue of involuntary infertility more pressing. If couples are urged to postpone or widely space pregnancies, it is imperative that they should be helped to achieve pregnancy when they so decide, in the more limited time they will have available' [35].

The Programme provided technical assistance to the WHO Department of Health Statistics and Information Systems for a process whose aim was to determine the burden, trends over time, and the relative impact of infertility on society. This process addressed infertility as a disability, as 'an impairment which is a problem in body function or structure'. A systematic research analysis defining trends in infertility since 1990 has been completed [unpubl. data], and a disability envelope for infertility revealed that the impact on society ranked high. Significantly, when the top 20 major global causes of disability were collated, infertility (arising from maternal sepsis and unsafe abortion practices) was ranked 8th overall, and 6th among all people under the age of 60 , with the majority of the burden in developing countries [37]. As stated in the preface written by Margaret Chan, Director General of the WHO, and Robert Zoellick, President of the World Bank, this report 'suggests steps for all stakeholders to create enabling environments ... create inclusive policies and programmes, and enforce new and existing standards and legislation, to the benefit of people with disabilities and the wider community.... Our driving vision is of an inclusive world in which we are all able to live a life of health, comfort, and dignity' [37].

Despite the increasing evidence of infertility's high impact on society, access to care and increasing its prioritization within the public health agenda remain fraught with barriers. Recognizing the importance of infertility on the global health stage, supported by fertility societies, such as ESHRE, ASRM, FIGO, IFFS, ISIVF, ISMAAR, the West African Society of Reproductive Medicine, GIERAF, or nonprofits, such as The Walking Egg or the Low Cost IVF Foundation, the WHO was able to continue to provide technical and advocacy presentations drawn from meeting outcomes, surveys, and the Disability Report, as well as opportunities to present on ethical issues. Issues surrounding fertility decision making and quality of life indicators were discussed together with the WHO at meetings held at or in collaboration with and supported through projects granted to Cardiff University, Wales; moreover, affordable IVF and laboratory solutions were discussed at the Genk Institute for Fertility Technology in Belgium, as well as the University of Colorado in Boulder, Col., USA, and the University of Geneva, Switzerland. 
In 2011, the Programme in collaboration with the Social Science Study Group of the ESHRE Task Force, together with the nonprofit The Walking Egg and the University of Amsterdam, The Netherlands, held a workshop in Belgium with predominant participation from researchers from developing countries and countries in transition with an aim to report on their studies which addressed barriers to access to infertility care and treatment, and to assess and report on the complications when attempting implementation (research or practice) of affordable infertility care [38].

The current lack of updated WHO global guidelines and the tools required for dissemination of best practices for affordable infertility care has, in itself, become a barrier for access to care, and there are frequent requests for these documents from Member States. With support and representation from ASRM, ESHRE, FIGO, ICMART, and IFFS, the Programme, in collaboration with 36 experts from around the world who donated their time and expertise, has begun the process of updating the WHO global guidelines for infertility diagnosis, management, and interventions for treatment. A critical scoping was begun with initial and prioritized areas tentatively identified. The Programme initiated an innovative training/ consultation mechanism with support from the Graduate Foundation for Medical Education and Research (GFMER) which ensures strong developing and transitional country input throughout the process. A gift of visual art specifically created for this initiative was donated to the HRP Programme from the nonprofit The Walking Egg.

\section{Partnering with a Promising Future}

The previous Programme Task Force had established and helped to support a significant number of official WHO collaborating centers (WHO-CCs) with joint plans of work in research, covering infertility and low-fertility issues. Currently, two WHO-CCs remain and finance their research on infertility: (1) in Kenya, the Institute for Primate Research (IPR) addresses basic science aspects of ART and endometriosis. In 2010, the Programme participated in a symposium in celebration of the 50th anniversary of the IRP and presented on topics linked to ethical considerations in NHP research. (2) In Belgium, the work in Rwanda of the University of Ghent, under the direction of Marleen Temmerman, addressing issues associated with infertility (e.g. intimate partner violence, or HIV) has just been completed [39] and was presented in the workshop jointly sponsored by the Programme [38]. A third WHO-CC in Germany, at the University of Münster, has been supported by the Programme for work linked to male fertility issues - research to generate the evidence base to support as well as to assist with the revision of the Semen Manual.

In addition to the recent historical collaborative events reported, the Programme recently renewed or instated (a minimum 4-year process) plans for work with three NGOs in official relations with the WHO: FIGO, IFFS, and ICMART (new). The planned joint activities have resulted in regional and national workshops, as well as the generation of critical tools for stakeholders to increase access and/or assist with monitoring and surveillance of infertility activities, e.g. the ICMART Tool Box for monitoring ART practices [40], the IFFS Surveillance Reports [41], and UIT-IFFS Resource Centre [42], as well as the FIGO Reproductive Medicine Committee's FIGO Fertility Tool Box [43].

Our partners (individuals as well as public and private partnerships) in support of the Programme have been able to keep a vision alive and provide contributions to reproduction research innovations in order to address the fertility needs of all peoples throughout their life cycle, within the realm of the global public health arena, dealing with reproductive as well as maternal and child health outcomes. Marleen Temmerman, the new director of the WHO Department of RHR and the HRP Special Programme, has recognized the field of infertility as a neglected area of reproductive health, as presented in her interview [44], and there is optimism that projects addressing infertility diagnosis, management, prevention, and treatment as well as psychosocial, ethical, legal, and socioeconomic aspects of providing infertility care and treatment will return more prominently to HRP's core research agenda and will again become one of the Programme's health priorities of focus.

The WHO would like to thank the many contributors to the Programme's infertility projects and activities, without whom the advances made or begun on behalf of the infertile worldwide would not have been possible. For reasons of writing a concise history, a description of the important contributions each individual has made is beyond the scope of this article. The author would like to apologize for the many contributions or activities which, due to space constraints, have been omitted or have been only briefly mentioned or referenced. 


\section{References}

1 Eighteenth World Health Assembly. 1. Resolutions and decisions, annexes. World Health Assembly 18.49. Official records of the World Health Organization, No 143, 35, 1965.

2 World Health Organization: Twenty-First World Health Assembly. 1. Resolutions and decisions, annexes. Health aspects of population dynamics. World Health Assembly 21.43. Official records of the World Health Organization, No 168, 21-22, 1968.

3 World Health Organization: Developments in fertility control: report of a WHO Scientific Group. WHO Technical Report Series 424. Geneva, WHO, 1969.

4 Bustreo F, Mbizvo M: Human Reproductive Program: the first partnership for health and development at the WHO. Gynecol Obstet Invest 2012;74:181-184.

5 World Health Organization, Diczfalusy E, Standley CC (eds): The Use of Non-Human Primates in Research on Human Reproduction: a symposium organized by the WHO in collaboration with the Ministry of Health of the USSR. Stockholm, WHO Research and Training Centre on Human Reproduction, Karolinska Institutet, 1972.

6 World Health Organization: Epidemiology of infertility: report of a WHO Scientific Group. WHO Technical Report Series 582. Geneva, WHO, 1975.

7 World Health Organization, Rowe PJ, Raharinosy-Ramarozaka SR (eds): Diagnosis and Treatment of Infertility: WHO Workshop, Nairobi, 1979. Reading, Pitman Press Limited, 1980.

8 A joint report by the director-general of the World Health Organization and the executive director of the United Nations Children's Fund: primary health care - report of the International Conference on Primary Health Care, Alma Atta, USSR, 6-12 September 1978. Geneva, WHO, 1978.

$\checkmark 9$ Cates W, Farley TM, Rowe PJ: Worldwide patterns of infertility: is Africa different? Lancet 1985;2:596-598.

10 World Health Organization, Task Force on the Diagnosis and Treatment of Infertility: Infections, pregnancies, and infertility: perspectives on prevention. Fertil Steril 1987;47: 964-968.

11 White R, Zaba B, Boerma JT, Blacker J: Modeling the dramatic decline of primary infertility in Sub-Saharan Africa. Reprod Health Matters 1999;7:13.

12 The Nobel Prize in Physiology or Medicine, 2010: Robert G. Edwards. http://www.nobelprize.org/nobel_prizes/medicine/laureates/2010/press.html.

13 Giwa-Osagie OF, Ogunyemi D, Emuveyan EE: Etiological classification and social medical characteristics of infertility: 250 couples. Int J Fertil 1984;147:964.
14 World Health Organization, Rowe PJ, Vikhlyaeva EM (eds): Diagnosis and Treatment of Infertility. Bern, Hans Huber, 1988.

15 World Health Organization: Recent advances in medically assisted conception. WHO Technical Report Series 820. Geneva, WHO, 1992.

16 World Health Organization, Rowe PJ, Comhaire FH, Hargreave TB, Mellows HJ (eds): WHO Manual for the Standardized Investigation and Diagnosis of the Infertile Couple. Cambridge, Cambridge University Press, 1993.

17 World Health Organization: WHO Laboratory Manual for the Examination of Human Semen and Sperm-Cervical Mucus Interaction. Singapore, Press Concern, 1980.

18 World Health Organization, Rowe $\mathrm{PH}$, Comhaire FH, Hargreave TB, Mahmoud AMA (eds): Manual for the Standardized Investigation, Diagnosis and Management of the Infertile Man. Cambridge, Cambridge University Press, 2000.

19 World Health Organization: WHO Laboratory Manual for the Examination of Human Semen and Sperm-Cervical Mucus Interaction, ed 4. Cambridge, Cambridge University Press, 1999.

20 Lozano EA, Padrón D, Rubén S, Seuc AJ: Gonadotropinas y sexoesteroides en mujeres posmenopáusicas del círculo de abuelos. Rev Cubana Med Gen Integr 1997;13:489-493.

21 Joint South African and WHO Reproductive Task Force under the auspices of the Ministry of Health Family Planning Assessment, October 1994.

22 Thomas C: Planned Parenthood Association of South Africa and AVSSA: infertility - a challenge to South African health services. Sex Reprod Health Bull 1995;1:7-8.

23 United Nations Commission on Human Rights Resolution 2003/28, preamble and para. 6.

24 The World Health Organization, Vayena E, Rowe PJ, Griffin PD (eds): Current Practices and Controversies in Assisted Reproduction: Report of a WHO Meeting 'Medical, Ethical and Social Aspects of Assisted Reproduction'. Department of Reproductive Health and Research, Family and Community Health. Geneva, WHO, 2002.

25 Vayena E, Rowe PJ, Peterson HB: Assisted reproductive technology in developing countries: why should we care? Fertil Steril 2002; 78:13-15.

26 Sekadde-Kigondu CB, Chikamata DM, Franken DR: Infertility Management in Africa and Eastern Mediterranean Regions: A World Health Organization Workshop, Nairobi, Kenya, 4-8 February. Nairobi, Noni Publicity, 2005.
27 Rutstein SO, Shah IH: Infecundity, Infertility, and Childlessness in Developing Countries. DHS Comparative Reports. Calverton, ORC Macro, WHO, 2004.

28 World Health Organization, Department of Reproductive Health and Research including UNDP/ UNFPA/ WHO/ World Bank Special Programme of Research: Reproductive Health Strategy to accelerate progress towards the attainment of international development goals and targets. 57th World Health Assembly 2004, Geneva, WHO, 2004.

29 ESHRE Monographs, Ombelet W, Devroey P, Gianaroli L, Velde E (eds): ESHRE Special Task Force on: Developing Countries and Infertility. Oxford, Oxford University Press, 2008.

30 World Health Organization, Vayena E, Rowe PJ, Griffin PD (eds): Current Practices and Controversies in Assisted Reproduction: Report of a WHO Meeting 'Medical, Ethical and Social Aspects of Assisted Reproduction'. Department of Reproductive Health and Research, Family and Community Health. Geneva, WHO, 2002, pp xix-xxi.

- 31 Zegers-Hochschild F, Adamson GD, de Mouzon J, Ishihara O, Mansour R, Nygren K, Sullivan E, Van der Poel S, on behalf of the International Committee Monitoring Assisted Reproductive Technologies and the World Health Organization: The International Committee for Monitoring Assisted Reproductive Technology (ICMART) and the World Health Organization (WHO) Revised Glossary on ART Terminology. Hum Reprod 2009;24:2683-2687.

32 Chou D, Tuncalp O, Hotamisligil S, Norman J, Say L, Genito-Urinary Reproductive Medicine TAG: Steps through the revision process of reproductive health sections of ICD11. Gynecol Obstet Invest 2012;74:228-232.

-33 De Neubourg D, van Duijnhoven NT, Nelen WLDM, D'Hooghe TM: Dutch translation of the ICMART-WHO Revised Glossary on ART Terminology: Nederlandse vertaling van de ICMART-WHO herziene woordenlijst over ART terminologie. Gynecol Obstet Invest 2012;74:233-248.

34 Program on International Health and $\mathrm{Hu}-$ man Rights, World Health Organization with the support of Harvard Centre for Population and Development Studies, Elizabeth Glaser Pediatric AIDS Foundation, David Rockefeller Center for Latin American Studies Harvard University, and the National Institutes of Health: The Pregnancy Intentions of HIV-Positive Women: Forwarding the Research Agenda - Conference Report, Boston, 17-19 March, 2010.

35 World Health Organization: Mother or nothing: the agony of infertility. Bull World Health Organ 2010;88:881-882. 
36 World Health Organization: Maasai couples seek safer solutions to infertility. Bull World Health Organ 2010;88:885-886.

37 World Health Organization, The World Bank: World report on disability. Geneva, WHO, 2011.

38 Social Science Study Group of the ESHRE Special Task Force 'Developing Countries and Infertility' in cooperation with The Walking Egg npo, the University of Amsterdam, and the WHO, Gerrits T, Ombelet W, Van Balen F, Vanderpoel S (eds): Biomedical Infertility Care in Poor Resource Countries:
Barriers, Access and Ethics. Facts, Views and Vision, Issues in Obstetrics, Gynaecology and Reproductive Health, 2012.

39 Dhont N: Clinical, Epidemiological and Socio-Cultural Aspects of Infertility in Resource-Poor Settings: Evidence from Rwanda; doctoral thesis, Ghent University, and Amsterdam University, 2011.

40 ICMART Tool Box for ART Data Collection. http://www.icmartivf.org/toolbox/toolboxmain.html.

41 Jones HW, Cooke I, Kempers R, Brinsden P, Saunders D: International Federation of Fer- tility Societies Surveillance 2010: preface. Fertil Steril 2011;95:491.

42 IFFS-UIT Resource Center. http://www.iffsuit.com/.

43 FIGO Fertility Tool Box. http://www.jsog. or.jp/news/pdf/October_2011_FIGO_newsletter.pdf.

44 D'Hooghe TM: Interview with Prof. Dr. Marleen Temmerman, new director of the WHO Department of Reproductive Health and Research, assuming office on October 15, 2012. Gynecol Obstet Invest 2012;74:187189 